\title{
Research Article \\ Improving 2D-Log-Number-System Representations by Use of an Optimal Base
}

\author{
Roberto Muscedere \\ Electrical and Computer Engineering Department, University of Windsor, Windsor, ON, Canada N9B3P4 \\ Correspondence should be addressed to Roberto Muscedere, rmusced@uwindsor.ca
}

Received 10 April 2008; Accepted 20 June 2008

Recommended by Ulrich Heute

The 2-dimensional logarithmic number system (2DLNS), a subset of the multi-DLNS (MDLNS), which has similar properties to the classical Logarithmic Number System (LNS), provides more degrees of freedom than the LNS by virtue of having two orthogonal bases and has the ability to use multiple 2DLNS components, or digits. The second base in 2DLNS can be adjusted to improve the representation space for particular applications; the difficulty is selecting such a base. This paper demonstrates how an optimal second base can considerably reduce the complexity of the system while significantly improving the representation space for application specific designs. The method presented here maps a specific set of numbers into the 2DLNS domain as efficiently as possible; a process that can be applied to any application. By moving from a two-bit sign to a one-bit sign, the computation time of the optimal base is halved, and the critical paths in existing architectures are reduced.

Copyright (c) 2008 Roberto Muscedere. This is an open access article distributed under the Creative Commons Attribution License, which permits unrestricted use, distribution, and reproduction in any medium, provided the original work is properly cited.

\section{INTRODUCTION}

The 2-dimensional logarithmic number system (2DLNS), a subset of the multi-DLNS (MDLNS) [1], a generalization of the index calculus introduced into the double-base number system (DBNS) [2, 3], uses 2 orthogonal bases (of which the first is 2) and has similar properties to the logarithmic number system (LNS) $[4,5]$. The 2DLNS has found initial applications in the implementation of special digital signal processing systems, where the operation on orthogonal bases greatly reduces both the hardware and the connectivity of the architecture. As with the LNS, some operations such as multiplication and division are relatively easy whereas operations of addition, subtraction, and conversion to standard representations are difficult. Current 2DLNS systems utilize architectures which favor any multiplication $[1,3,6]$ (or division) but try to minimize any use of addition or subtraction as they are considered costly functions since they traditionally require large lookup tables (LUTs). One of the most popular 2DLNS architectures is the inner product computational processor which performs multiplication in the 2DLNS domain, converts to the binary domain, and then accumulates the result. This conversion requires LUTs whose size is dictated by the range of the second-base exponent.

This paper demonstrates how an optimal base can significantly reduce the range on the second-base exponent and therefore the hardware needed for this and potentially future 2DLNS architectures. This reduction makes these types of architectures more competitive with existing systems based on fixed-point and floating-point binary as well as those based on LNS. We also show that migrating from a two-bit sign system to a one-bit sign system can half the computation time of determining the optimal base as well as reduce the critical paths of an established architecture.

\section{BACKGROUND}

\subsection{Multi-digit 2DLNS representation}

A 2DLNS representation is a subset of the MDLNS with only two bases (an $n$-digit 2DLNS representation). The first base is usually referred to as the binary base while the other is the nonbinary base or second base. We will assume that the exponents have a predefined finite precision equivalent to limiting the number of bits of precision in a classic LNS. The 
simplified representation of a value, $x$, as an $n$-digit 2DLNS is shown as follows:

$$
x=\sum_{i=1}^{n} s_{i} \cdot 2^{a_{i}} \cdot D^{b_{i}}+\varepsilon .
$$

A sign, $s_{i}$, is required as the exponents cannot influence the sign of the representation. $s_{i}$ is typically -1 or 1 but the case $s_{i}=0$ is required when either the number of digits required to represent $x$ is less than $n$, or the special case when $x=0$. The second base, $D$, is our target for optimization. It should be chosen such that it is relatively prime to 2, but it does not necessarily need to be an integer especially in signal processing applications. This extension can vastly increase the chance to obtain an extremely good representation of a particular set of numbers with very small exponents especially with two or more digits. The exponents are integers with a constrained precision. $R$ is the bit-width of the second-base exponent, such that $b_{i}=\left\{-2^{R-1}, \ldots, 2^{R-1}-\right.$ $1\}$. This value directly affects the complexity of the MDLNS system. We will also define $B$ as the bit-width of the binary exponent, such that $a_{i}=\left\{-2^{B-1}, \ldots, 2^{B-1}-1\right\}$. Later, when we look at a practical example, the resolution of these exponent ranges will be further refined as the full bit range will be rather excessive. Unlike $R, B$ does not directly effect the complexity of the system. We define these values since our 2DLNS system is to be realized in hardware. We also consider $\varepsilon$ as the error between the 2DLNS representation and the intended value of $x$.

\subsection{Single-digit $2 D L N S$ representation}

We start our discussion by examining the single-digit 2DLNS case. Setting $n=1$ in (1), we obtain the simplified singledigit 2DLNS representation as follows:

$$
x=s \cdot 2^{a} \cdot D^{b} .
$$

\subsection{Single-digit 2DLNS inner product computational unit}

Figure 1 shows the structure of the single-digit 2DLNS inner product computation unit (CU) from [3]. The multiplication is performed by small parallel adders for each of the operands base exponents (top of the figure). The output from the second-base adder is the address for an LUT or ROM which produces an equivalent floating point value for the product of the nonbinary bases (i.e., $D^{b_{1}+b_{2}} \approx 2^{\xi_{B}} \cdot \xi_{M}$ ). The base 2 exponents are added to that of the table to provide the appropriate correction to the subsequent barrel shifter (i.e., $2^{a_{1}+a_{2}} D^{b_{1}+b_{2}} \approx 2^{a_{1}+a_{2}+\xi_{B}} \cdot \xi_{M}$ ). This result may then be converted to a 2's complement representation, set to zero, or unmodified based on the product of the signs of the two inputs $(-1,0$, or 1 , resp.). The final result is then accumulated with a past result to form the total accumulation (i.e., $\left.y(n+1)=y(n)+2^{a_{1}+a_{2}+\xi_{B}} \cdot \xi_{M}\right)$.

This structure removes the difficult operation of addition/subtraction in 2DLNS by converting the product into binary for simpler accumulation. It is best for feedforward



FIGURE 1: One-digit 2DLNS inner product computational unit from [3].

architectures. We note that when the range of the secondbase exponent, $R$, of the 2DLNS representation is small (e.g., less than 4 bits), then these LUTs will be very small as well.

The structure can be extended to handle more bases by concatenating the output of each corresponding exponent adder to generate the appropriate address for the LUT. The penalty however is that every extra address bit doubles the LUT entries. The structure itself will be replicated depending on the number of digits. If both operands have the same number of digits, we can expect to have $n^{2}$ such units in an $n$-digit MDLNS. For a parallel system, these outputs could be summed at the end of the array using an adder tree for example. The biggest advantage of the use of more than one digit for the operands is that one can obtain extremely accurate representations with very small exponents on the second base. But the area cost increases as the number of computational channels required is increased to at least four.

\section{SELECTING AN OPTIMAL BASE}

\subsection{The impact of the second base on hardware}

A closer look into the architecture above shows that the LUT stores the floating-point-like representation of the powers of the second base $D$. The area complexity depends almost entirely on the size of the LUT which is determined by the range of the sum of the second base-exponents, $b_{1}$ and $b_{2}$. Our main goal in selecting the second base is to minimize, as much as possible, the size of the largest secondbase exponents used while maintaining the application constraints. The actual value of $D$ can be selected to optimize the implementation without changing the overall 
complexity of the architecture; in fact, as we will see, such an optimization offers a great potential for further reductions of the hardware complexity. Therefore, any value of $D$ will only change the contents of the LUT while the range of the second-base exponents is the only factor which influences the size of the LUT. The same can be said for the binary-toMDLNS converters found in [7]; their complexity is limited by this range as well as the number of digits.

\subsection{Defining a finite limit for the second base}

We can limit the potential range of what could be considered to be an optimal value by analyzing the unsigned single-digit representation as shown in (3),

$$
2^{a} D^{b}=2^{a-b}(2 D)^{b}=2^{a+b}\left(\frac{D}{2}\right)^{b} .
$$

This expression shows that we can multiply or divide the unknown base by any multiple of the first base there changing its exponent but not changing the computational result. This simple relationship implies a restriction on the range of values of an optimal base. For example, if our search was to begin at $D=3$, then it would be pointless to go outside of the range 3 to 6 as the results of the representation would simply repeat.

The relationship in (3) also shows that as the value of $D$ is divided by a multiple of 2 , the exponent of the first base will increase when $b$ is positive but decrease when $b$ is negative. A similar conclusion can be made for the case when $D$ is multiplied by a multiple of 2 . Therefore, some representations may have large values for the first base exponent, and some may have smaller values. For a hardware implementation, the bit-width of the first base exponent should be minimized while maintaining the selected representation space. We can determine the bit-width for the first base exponent by limiting our representation with (4),

$$
1 \leq 2^{a} D^{b}<2 .
$$

There is a unique first base exponent for every secondbase exponent. We continue by taking the logarithm of (4) as shown in (5),

$$
0 \leq a \ln (2)+b \ln (D)<\ln (2) .
$$

From (5), we obtain limits on the first base exponent, as shown in (6),

$$
-b \frac{\ln (D)}{\ln (2)} \leq a<1-b \frac{\ln (D)}{\ln (2)} .
$$

Since the range of $b$ is known, the value of $a$ can be found for all valid values of $b$. From this, the integer range of $a$ can be found from the maximum and minimum values of $b$. The binary word length of the usable 2DLNS range is added to the maximum integer range of $a$ to find the total range of $a$. For example, if $D=3$ and $b$ ranges from -4 to 3 ( 4 bits), then the range for the first base exponent will be between -4 and 7 for numbers between 1 and 2. If we wish to represent at most a 9bit integer, then we will require a range of $[-4,(7+9=16)]$ for the first base exponent, or 6 bits.
Using these relationships, we can potentially reduce the number of bits required to represent $a$. From (6), the range of $a$ depends on the factor $\ln (D) / \ln (2)$, where minimizing $\ln (D)$ results in a smaller bit-width on $a$. Since the factor has a denominator of $\ln (2)$, any integer multiple of 2 on $D$ will produce the same 2DLNS results. The function $\ln (D)$ will be minimized when $D$ is closest to 1 . The optimal range of $D$ can thus be found by relating $\ln (y)$ (which is $>1$ ) with $\ln (y / 2)$ (which is $<1$ ). Setting $\ln (y)=-\ln (y / 2)$, we obtain $y=\sqrt{2}$. Therefore, the optimal range of $D$ is between $\sqrt{2} / 2$ (or $1 / \sqrt{2}$ ) and $\sqrt{2}$. We now have established an optimal range for $D$ that will provide a minimal bit-width to represent the first base exponent, $a$ and eliminate base replication.

If we rework our previous example using $D=0.75$ (3 divided by 4 ) and set the range of $b$ to $[-4,3]$ (4 bits), the range for the first base exponent will be between -1 and 2 . To represent a maximum of a 9-bit integer, we will require a range of $[-1,(2+9=11)]$ for the first base exponent, or 5 bits. This is a saving of 1 bit from the previous example, where $D=3$, but with no change in the representation.

\subsection{Finding the optimal second base}

We have developed two methods for determining the optimal base for $m$ numbers in the set $x$. The first, an algorithmic approach, only applies to single-digit 2DLNS, and the second, a range search, applies to any number of digits.

\subsubsection{Algorithmic search}

Using the assumption that the optimal base represents one of the values in the given set $x$ with virtually no error $(\varepsilon \cong$ 0 ), then that optimal base can be found by solving the base from the single-digit unsigned 2DLNS expression as in the following:

$$
D=\sqrt[b]{\frac{x}{2^{a}}} \quad \text { or } \quad D=x^{1 / b} 2^{-a / b}
$$

This expression can be solved for every value in the set $x$ given the range on $b$ which depends on $R$ (i.e., $b=$ $\left.\left\{-2^{R-1}, \ldots,-1,1, \ldots, 2^{R-1}-1\right\}\right)$. Since any multiple of 2 on $D$ does not effect the 2DLNS representation, $a$ is limited by $b$, such that $a=\{-b+1, \ldots, b-1\}$. Although many solutions may exist depending on the value of $R$ and the number of values $x$, only the bases with the smallest errors will be finely adjusted until the final optimal base is found (see Section 3.3.3).

\subsubsection{Range search}

A second alternative is to perform a range search through all the possible real bases. We have already seen that the most efficient bases for hardware implementation lie in the range $[1 / \sqrt{2}, \sqrt{2}]$. This limitation offers a practical start and end point for a range search. Given an arbitrary second base, the program measures the error of mapping the given set $x$ into a multidigit 2DLNS representation. The possible representation methods can reflect those of hardware methods available such as the greedy/quick, high/low approximations 
[7] or a brute force approach. The program uses a dynamic step size which is continuously adjusted by analyzing the change in the mapping errors for a series of test points. This step size increases so long as the resulting errors are monotonically improving. If this is not the case, the program retraces and decreases this step size. When a better error is found it is added to a running list of optimal candidates. Using a dynamic step size is effective in finding optimal base candidates while also reducing the overall search time. Once the entire range has been processed, each element in this list is finely adjusted. Depending on the representation method selected and the range of $R$, this approach can generate fewer bases than the algorithmic method and therefore produce results in a shorter amount of time.

\subsubsection{Fine adjustment}

A fine adjustment is performed with the list of optimal candidates by progressively adding and subtracting smaller and smaller values. The performance of the software is further increased by using direct floating point (IEEE 64-bit) manipulation as well as minimizing conditional branches and expensive function calls. This approach drastically improves search times by initially performing a coarse search, by one of the methods above, and then a finer search near the selected optimum points.

\section{ONE-BIT SIGN ARCHITECTURE}

The data path of the 2DLNS processor (in Figure 1) is affected significantly by the signs of the operands. The required sign correction operation comes at a cost of additional logic and power. Thus far, a multidigit architecture would require additional processing to be performed after the 2DLNS processor, such as summing all the channels. It is possible to use the common one-bit sign binary representation for the intermediate results. We have therefore developed a new 2DLNS sign system to reduce the processing path of the 2DLNS inner product CU while producing a single sign-bit binary representation.

\subsection{Representation efficiency}

Our original 2DLNS notation uses two bits to represent the sign for each digit $(-1,0$, and 1$)$ however only three of four states are used, one of which (zero) only represents a single value. By using two bits for the sign, the efficiency of the representation is approximately 50 percent:

$$
\begin{aligned}
& \text { efficiency }_{\text {two-bit sign }} \\
& =\frac{\text { valid representations }}{\text { total possibilities }}=\frac{2^{1+B+R}+1}{2^{2+B+R}} \cong 0.5 .
\end{aligned}
$$

To improve this efficiency, we propose that only a single sign-bit is needed to represent the most common cases, that is, -1 and 1 . We then choose to represent zero by setting the second-base exponents to their most negative values (i.e., if the range is $[-4,3]$, then -4 is used to represent zero). This allows us to reduce the circuitry of the system while maintaining the independent processing paths of the exponents; this modification is easily integrated into the existing two-bit sign architecture. This special case for zero still leaves us with a significantly smaller unused representation space compared to the two-bit sign system. As $R$ increases, the valid representations ratio approaches 1 :

$$
\begin{aligned}
& \text { efficiency }_{\text {one-bit sign }} \\
& \qquad=1-\frac{\text { invalid representations }}{\text { total possibilities }}=1-\frac{2^{1+B}}{2^{1+B+R}}=\frac{2^{R}-1}{2^{R}} \longrightarrow 1 .
\end{aligned}
$$

With the one-bit sign system, the range of the second base changes to $b_{i}=\left\{-2^{R-1}+1, \ldots, 2^{R-1}-1\right\}$ with a special case of $b_{i}=-2^{R-1}$ representing zero.

\subsection{Effects on determining the optimal base}

Since the upper and lower bounds of the second-base exponent are equal in magnitude, this eliminates the need for any reciprocal computations in determining the optimal base (i.e., $D^{b}=1 / D^{-b}$ ) thus approximately halving the search time for both algorithms. For the algorithmic search, the possible range of $b$ is changed, such that $b=\left\{1, \ldots, 2^{R-1}-1\right\}$. For the range search approach the second base limits are now $([1 / \sqrt{2}, 1.0]$ or $[1.0, \sqrt{2}])$.

\subsection{Effects on hardware architecture}

By using the one sign-bit architecture, the word length for any 2DLNS representation is reduced by 1 bit per digit. Compared to the original CU, we can remove the sign corrector component (essentially a conditional 2's complement generator). The sign is calculated by simply XORing the two signs of the inputs. The output is now only an absolute binary representation which can easily be manipulated further with the sign bit depending on the number of digits (see Figure 2). The special zero case only needs to be handled by modifying the very small adders in the multiplication component; the representation of zero is now inside the table and therefore eliminates the conditional path.

To accumulate this result with any other value, we can use the generated sign bit to determine the proper operation of an addition/subtraction component (see Figure 3 ). The inclusion of a one-bit sign allows us to reduce the hardware and computational path by removing the zero/two's complement generator. The final adder/subtractor component itself is slightly larger than an adder, but with regards to the whole system, this architecture will consume less area and time.

In the case of a two-digit 2DLNS system, the accumulation of the four output channels can be simplified with the one-bit sign by using only 3 adder/subtractor components and simple logic to coordinate the proper series of operations (see Figure 4). The processing delay from the LUTs is only 3 arithmetic operations and the overall logic is also reduced since the 3 adder/subtractor components are smaller than the 3 separate adders and four 2's complement generator components present in the original CU. This approach was 


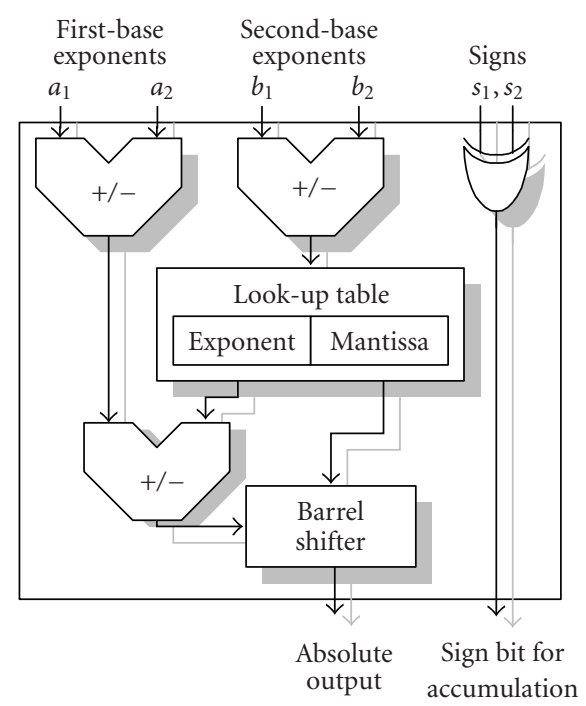

FIGURE 2: One-bit sign 2DLNS inner product computational unit.

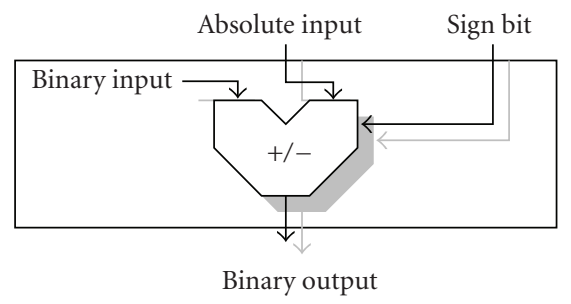

FIgURE 3: Single-digit one-bit sign accumulation component.

used in [6] and showed a 55\% saving in power as well as other improvements compared to the original design in [8]. Further hardware reductions can be made by ordering each 2DLNS processor in order of product magnitude. The resulting binary representation will be the largest for the first channel but will be decreased for each of the subsequent channels. If the range of both operands is known, the mantissa in the LUTs can be sized correctly as well as the subsequent adders.

\section{EXAMPLE FINITE IMPULSE RESPONSE FILTER}

To demonstrate how important it is to choose an optimum base, $D$, we provide the following example of a 47-tap lowpass FIR filter. There are many methods for designing digital filters, each of which prioritizes different output characteristics. In our case, we will use a simple set of characteristics which generalizes the problem so that the proposed method can be applied to any other application. For this example, we will minimize the passband ripple $(<0.01 \mathrm{~dB})$, maximize the stop band attenuation, and maintain linear phase (the coefficients will be mirrored in order to guarantee linear phase). To further reduce the complexity of this problem, we will first generate the filter coefficients by using classical design techniques. Ideally, using floating point values, we obtain a passband ripple of $0.0008 \mathrm{~dB}$ and a stop band attenuation of $81.1030 \mathrm{~dB}$ (see Figure 5).

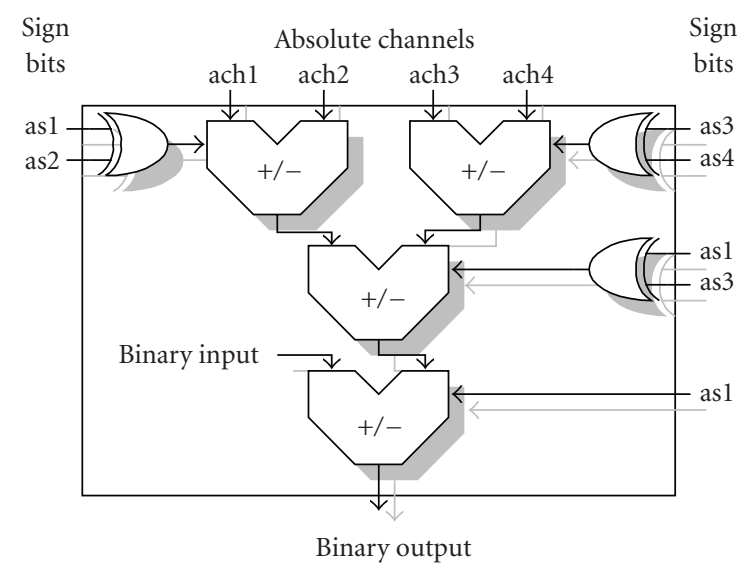

FIgURE 4: Two-digit one-bit sign accumulation component.



FIGURE 5: Magnitude response of the 47-tap FIR filter; $\omega_{p}=0.4$ and $\omega_{s}=0.6$

We will compare the results between a standard base of 3 as it has been used often in other published work. We could use any arbitrary base and the results would be similar. Once we have the real FIR filter coefficients, we then map them into a 2DLNS representation. If our mapping is poor, we can expect equally poor stop band attenuation as well as passband ripple, whereas a more accurate mapping will result in better filter performance. We choose not to calculate the filter's performance during the calculation of the optimal base, but rather the absolute error in the mapping itself. This improves the performance of the optimal base calculation and allows the process to be used with any filter design techniques or even for other applications entirely. Note that we do not impose restrictions on the size of the binary exponents as they have very little contribution to the overall complexity of the architecture. We require, however to know what their range will be for hardware implementation. An FIR filter basically multiplies a series of "data" values (from some external source) to a set of filter "coefficients" to generate an "output;" these terms will be used throughout the rest of this design. Since 


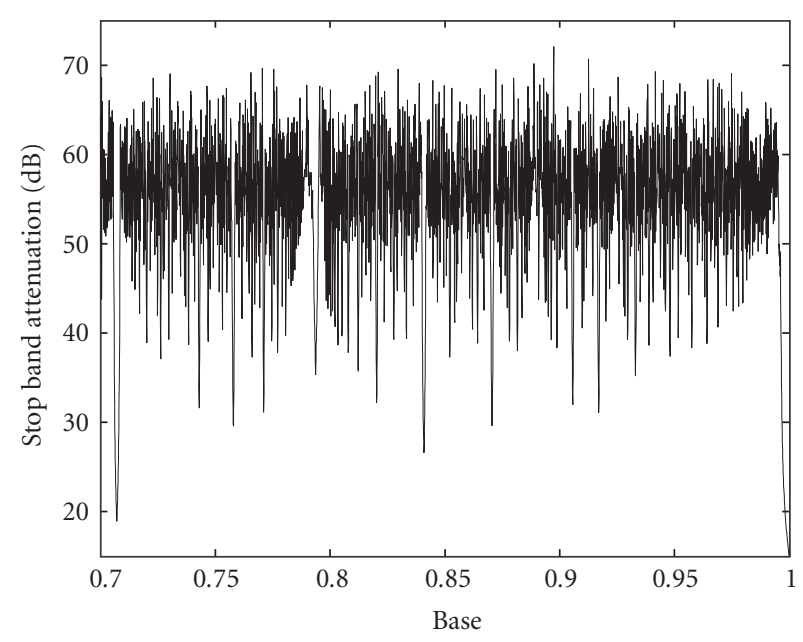

FIGURE 6: Stop band attenuation for bases 0.7071 to 1.0000 for $r_{c}=$ 63; worst case is $15.0285 \mathrm{~dB}$, best case is $72.0858 \mathrm{~dB}$.

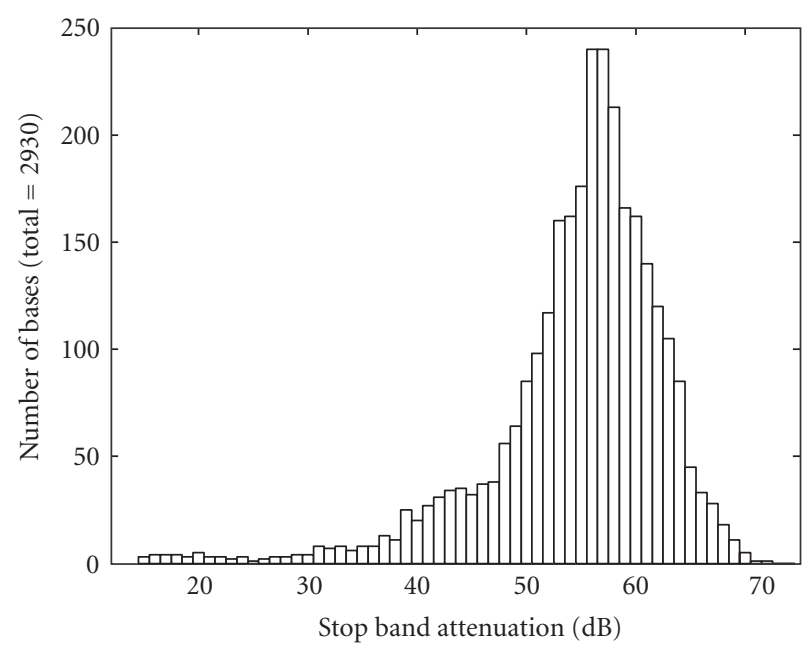

Figure 7: Histogram of stop band attenuation for bases 0.7071 to 1.0000 .

we are discussing the 2DLNS representations of the data, coefficient and product values in the same system, we will refer to their exponents as $\left(a_{d}, b_{d}\right),\left(a_{c}, b_{c}\right)$, and $\left(a_{o}, b_{o}\right)$, respectively. We will also compensate for a finer resolution on the multiplicands such that $b_{d}=\left\{-r_{d}, \ldots, r_{d}\right\}$ and $b_{c}=$ $\left\{-r_{c}, \ldots, r_{c}\right\}$ resulting in a product where $r_{o}=r_{d}+r_{c}$ and $b_{o}=\left\{-r_{o}, \ldots, r_{o}\right\}$. The range of the products second-base exponent, $b_{o}$, will dictate the complexity of the system.

To demonstrate how any arbitrary base can affect the filters performance, we have mapped the coefficients into a single-digit 2DLNS using bases between $1 / \sqrt{2}$ and 1.0 (in increments of 0.0001) and plotted the resulting stop band attenuation in Figure 6 for $r_{c}=63$.

This figure clearly shows that there is no obvious correlation between the filter's performance and the choice of the second base; in fact it appears random. The same can be said for the passband ripple. We can also examine these results in the form of a histogram as in Figure 7.
The low values of the stop band attenuation are a result of bases very close to 1.0 (where, as the exponent increases, the normalized representation approaches 1 ). The average is $54.6721 \mathrm{~dB}$, but for a base of 3 (or 0.75 ) it is $61.0460 \mathrm{~dB}$; which is better than the average results. Even though, our sample size for this test is small (2930 values), it is reasonable to assume that any arbitrary base will not give the best 2DLNS representational performance. The best base in this case is 0.8974 with a stop band attenuation of $72.0858 \mathrm{~dB}$. This is a good result but it is possible to achieve better without testing the filter's performance for every possible base.

\subsection{Optimizing the base through analysis of the coefficients}

Generally the signal samples or input "data" are large in magnitude and in order to accommodate for this, we will need to use two or more digits for their representation. If the input data was relatively small, we could use a one-digit representation, however we would expect some quantization. For our example, we will use a two-digit representation as the intended input range is larger ( -32768 to 32767 or 16 bits).

\subsubsection{Single-digit coefficients}

In [9], the typical distribution of the coefficients of many different filters was found to be a Gaussian-like function centered on zero. Such a coefficient distribution is better represented by a logarithmic-like number system (such as the LNS or 2DLNS) rather than a linear number representation (such as binary). Therefore, we should be able to obtain very good single-digit approximations in the 2DLNS by making use of a carefully calculated second base. Since the data representation uses two digits, the resulting system will consist of only two computational channels. We will also consider a two-digit 2DLNS representation with four channels later.

A comparison of the frequency response for a wide range of exponent ranges (or various values of $r_{c}$ ) for the example filter is shown in Table 1. We compare the passband ripple and stop band attenuation within a system with the second base of 3 and an optimal base. The optimal base is truncated to 6 decimal digits for presentation, however, the number of decimal digits is computed up to 15 (IEEE 64-bit floating point) and may be very necessary when the exponents on the base are large.

The table shows that as $r_{c}$ increases, we can save up to two bits on the second-base exponent by using an optimal second base rather than 3 . The size of the second-base exponent plays an important role in the size of the hardware due to the required LUTs; any 1-bit increase to any nonbinary base exponent doubles the LUT size, whereas an increase in the binary exponent adds minimal hardware. Any change to the second base, including real numbers has no impact on the structure of the hardware. Therefore, hardware designed for a second base of 3 is easily converted to use the optimal base as we are only changing the contents of the tables and not 
TABLE 1: Filter performance for ternary and optimal base (single-digit).

\begin{tabular}{|c|c|c|c|c|c|}
\hline \multirow{2}{*}{$r_{c}$} & \multicolumn{2}{|c|}{ Base 3} & \multicolumn{2}{|c|}{ Optimal base } & \multirow[b]{2}{*}{ Base } \\
\hline & Passband ripple $(\mathrm{dB})$ & Stop band attenuation $(\mathrm{dB})$ & Passband ripple (dB) & Stop band attenuation $(\mathrm{dB})$ & \\
\hline 1 & 0.5147 & 24.2840 & 0.1498 & 35.3497 & 0.793123 \\
\hline 3 & 0.2644 & 30.1926 & 0.0518 & 44.5401 & 0.889553 \\
\hline 7 & 0.0526 & 44.3046 & 0.0202 & 52.5938 & 0.888477 \\
\hline 15 & 0.0195 & 52.8771 & 0.0053 & 64.4114 & 0.897501 \\
\hline 31 & 0.0131 & 56.5177 & 0.0031 & 68.5123 & 0.897504 \\
\hline 63 & 0.0079 & 61.0460 & 0.0014 & 74.6231 & 0.788051 \\
\hline 127 & 0.0034 & 68.7088 & 0.0010 & 77.6842 & 0.908324 \\
\hline 255 & 0.0020 & 73.4312 & 0.0010 & 80.0042 & 0.876152 \\
\hline
\end{tabular}

TABLE 2: Filter performance for ternary and optimal base (two-digit).

\begin{tabular}{|c|c|c|c|c|c|}
\hline \multirow{2}{*}{$r_{c}$} & \multicolumn{2}{|c|}{ Base 3} & \multicolumn{2}{|c|}{ Optimal base } & \multirow[b]{2}{*}{ Base } \\
\hline & Passband ripple $(\mathrm{dB})$ & Stop band attenuation $(\mathrm{dB})$ & Passband ripple $(\mathrm{dB})$ & Stop band attenuation $(\mathrm{dB})$ & \\
\hline 1 & 0.0314 & 48.8313 & 0.0013 & 76.8648 & 0.757910 \\
\hline 2 & 0.0042 & 66.4051 & 0.0010 & 78.6542 & 0.899757 \\
\hline 3 & 0.0031 & 68.9081 & 0.0009 & 80.0583 & 0.957323 \\
\hline 4 & 0.0020 & 73.0290 & 0.0008 & 80.3113 & 0.789982 \\
\hline 5 & 0.0016 & 74.9437 & 0.0008 & 80.9408 & 0.814313 \\
\hline 6 & 0.0016 & 74.7669 & 0.0008 & 81.0408 & 0.919486 \\
\hline 7 & 0.0015 & 75.4867 & 0.0008 & 81.0342 & 0.885619 \\
\hline
\end{tabular}

their dimensions. In this case a two-bit reduction translates to a $4 \mathrm{X}$ area saving per LUT or CU.

\subsubsection{Two-digit coefficients}

We will continue using a two-digit representation for the signal and now use a two-digit representation for the coefficients. This will result in 4 parallel computational units. The method for generating these representations is via a brute force approach where effectively all possible representations are generated and the one with the least error is chosen. This method is not applicable to hardware as it is assumed; the coefficients will be generated offline. This approach was taken in [8] to improve 8 separate FIR filters in a filter bank application. Another comparison of the frequency response for various values of $r_{c}$ is shown for the two-digit coefficients in Table 2.

We stop at $r_{c}=7$ as the results are approaching near ideal. Again the use of an optimal second base offers the same stop band attenuation as with a second base of 3 but with two fewer bits. This saving is important as the CU in this case is duplicated four times.

\subsubsection{Comparison of single and two-digit coefficients}

In order for a one-digit 2DLNS to achieve $80 \mathrm{~dB}$ stop band attenuation, we need to use 9 bits $\left(r_{c}=255\right)$ for the second-base exponents and, correspondingly, we require an LUT with 512 entries for each CU (two for a parallel implementation). For a two-digit 2DLNS, we only need 3 bits $\left(r_{c}=3\right)$ to represent the second-base exponents therefore requiring an LUT with 8 entries for each CU (four for a parallel implementation). The two-digit coefficient system appears to be favorable as the LUTs are smaller; however, there is some additional overhead in the accumulation circuit for all the channels. It is also very important to note that this entire 4-channel architecture is multiplier-free as it consists only of small adders and very small LUTs.

\subsubsection{Effects on the two-digit data}

Clearly the choice of the second base has a significant effect on the performance of the filter. However, in order to use this representation effectively, we have to apply the same second base to the data representation or input signal as well in order for the 2DLNS arithmetic to operate properly. In the case of filter design, our optimal base is selected by the filter's performance which we can relate back to the quality of the mapping. In the case of data, specifically integers, we do not necessarily require perfect mapping but only errorfree representations where, from (1), $\varepsilon$ is less than half a bit or 0.5 [1]. Table 3 shows the range of $r_{d}$ for a $0 \%, 1 \%, 5 \%$, and $10 \%$ nonerror-free representations with a base of 3 and the optimal bases from Tables 1 and 2, respectively.

We can see that the optimal base for the best filter performance is not ideal for data mapping as $r_{d}$, on average, must be in the hundreds. When applying the optimal base to the coefficients, the performance increases as $r_{c}$ increases. There is no correlation here as the base was chosen only for optimal mapping of the coefficients. The case where $r_{d}=886$ in particular is unusual as this base produces bitstreams with long sequences of ones or zeros when the exponent exceeds 1 . 
TABLE 3: Data representation performance for various bases

\begin{tabular}{|c|c|c|c|c|c|c|c|}
\hline \multirow{2}{*}{ Base } & \multirow{2}{*}{$\begin{array}{c}\text { Error free } \\
r_{d}\end{array}$} & \multicolumn{2}{|c|}{$1 \%$ nonerror-free } & \multicolumn{2}{|c|}{$5 \%$ nonerror-free } & \multicolumn{2}{|c|}{$10 \%$ nonerror-free } \\
\hline & & $r_{d}$ & Worst $\varepsilon$ & $r_{d}$ & Worst $\varepsilon$ & $r_{d}$ & Worst $\varepsilon$. \\
\hline 3.000000 & 93 & 51 & 0.898 & 28 & 1.272 & 24 & 2.533 \\
\hline 0.793123 & 147 & 131 & 1.867 & 113 & 17.349 & 101 & 28.392 \\
\hline 0.889553 & 71 & 36 & 1.213 & 32 & 4.452 & 30 & 5.976 \\
\hline 0.888477 & 70 & 48 & 1.002 & 35 & 1.872 & 27 & 2.009 \\
\hline 0.897501 & 59 & 44 & 1.290 & 34 & 2.435 & 28 & 2.438 \\
\hline 0.897504 & 54 & 45 & 1.330 & 35 & 2.437 & 29 & 2.522 \\
\hline 0.788051 & 105 & 74 & 1.189 & 55 & 1.858 & 42 & 2.308 \\
\hline 0.908324 & 66 & 47 & 1.364 & 36 & 1.720 & 29 & 2.121 \\
\hline 0.876152 & 74 & 59 & 1.511 & 46 & 2.763 & 39 & 4.009 \\
\hline 0.757910 & 886 & 734 & 6.002 & 566 & 18.941 & 496 & 25.501 \\
\hline 0.899757 & 52 & 40 & 0.994 & 28 & 1.605 & 24 & 2.476 \\
\hline 0.957323 & 67 & 54 & 1.810 & 45 & 3.238 & 39 & 3.678 \\
\hline 0.789982 & 70 & 45 & 1.235 & 29 & 1.414 & 23 & 2.565 \\
\hline 0.814313 & 270 & 191 & 1.252 & 134 & 2.206 & 106 & 2.944 \\
\hline 0.919486 & 95 & 70 & 1.106 & 50 & 1.707 & 39 & 2.636 \\
\hline 0.885619 & 48 & 40 & 1.442 & 30 & 1.545 & 25 & 2.751 \\
\hline
\end{tabular}

\subsection{Optimizing the base through analysis of the data}

We have seen how applying an optimal base to the coefficients of a digital filter can significantly increase the accuracy of the 2DLNS representation. This same improvement can be seen when applied to the input data of the filter. For the case of a 16-bit signed input, from -32768 to 32767 , we require $r_{d}=39$ in order to achieve a completely error-free mapping using the high/low method [7] (the only published realtime binary to MDLNS conversion circuit). For particular applications however, a complete error-free mapping may not be necessary. Table 4 summarizes different choices of $r_{d}$ for nonerror-free integer mappings.

The trend of the number of nonerror-free representations follows an exponential decay as $r_{d}$ increases. From the optimal base calculations of the coefficients (see Table 3), we have the smallest $r_{d}$ of 36 with $1 \%$ nonerror-free representation but with a worst case error of 4.452 . The next smallest $r_{d}$ of 40 offers a worst case error of only 0.994 . Both cases require $r_{d}$ to be increased by more than $33 \%$ to achieve an error-free representation. When optimizing the base for the data representation, we can select $r_{d}=32$ to achieve less than $1 \%$ nonerror-free representation with a worst case $\varepsilon$ of 0.772 . This is comparable to $r_{d}=40$ in Table 3 but with a $25 \%$ reduction in the exponent range as well as the LUT entries. This approach was used in [6] so that the filter coefficients could be changed by mapping them into the optimal base selected for the data representation. This required a larger $r_{c}$ to improve the filter performance, but allowed the coefficients to be runtime loaded.

\subsection{Optimizing the base through analysis of both the coefficients and data}

We have so far seen that an optimal base can improve the coefficient or data representations of a 2DLNS filter architecture without changing the range of the exponents. Again, the 2DLNS arithmetic will not operate correctly unless both bases are the same. In each case the selection of one base severely impacts the other's representation. To remedy this, we have modified the optimal base software to target two separate scenarios. This is done by optimizing the two independent sets of values and minimizing the product of their errors.

\subsubsection{Single-digit coefficients and two-digit data}

For our example of an FIR filter, the data is represented with two-digit 2DLNS (using the high/low method) and the coefficients with a single-digit (later, two-digit brute-force method). Since the range of $r_{c}$ must be large for the singledigit coefficients to obtain good filter performance, we will also target an error-free data mapping as we can expect that $r_{d}$ will be close to 39 . Through experimenting with different variations of $r_{d}$, it was found that $r_{d}$ would have to be 42 in order to produce an error-free data representation. To maximize the data path utilization for $r_{o}$, the remaining bits are used to specify $r_{c}$; this technique has been virtually used in every DBNS/MDLNS paper to date. Table 5 shows the best results of the optimal base calculations for $8(42+85=127)$ and $9(42+213=255)$ bits. The resulting passband ripple is no longer presented on this or subsequent tables as it is always below the specification of $0.01 \mathrm{~dB}$. The bolded values in the table indicate the best result for the selected attribute. A bolded base is the author's choice for best stop band attenuation, nonerror-free representations, and the worst case error.

\subsubsection{Comparison to the individual optimal base}

Comparing the filter performance results of Tables 1 to 5 , we can see approximately a $2 \mathrm{~dB}$ reduction in the stop 
TABLE 4: Data representation performance for optimal bases.

\begin{tabular}{|c|c|c|c|c|}
\hline Base & $r_{d}$ & Nonerror-free representations & Worst $\varepsilon$ & $\%$ Nonerror-free \\
\hline 0.810537 & 16 & 16472 & 2.933 & 25.13 \\
\hline 0.853451 & 17 & 14296 & 2.857 & 21.81 \\
\hline 0.844819 & 18 & 12406 & 2.362 & 18.93 \\
\hline 0.867483 & 19 & 10926 & 2.156 & 16.67 \\
\hline 0.776015 & 20 & 9238 & 1.991 & 14.10 \\
\hline 0.797969 & 21 & 7836 & 1.717 & 11.96 \\
\hline 0.769616 & 22 & 6630 & 1.598 & 10.12 \\
\hline 0.915321 & 23 & 5566 & 1.540 & 8.49 \\
\hline 0.855890 & 24 & 4594 & 1.333 & 7.01 \\
\hline 0.838039 & 25 & 3746 & 1.343 & 5.72 \\
\hline 0.987020 & 26 & 3020 & 1.231 & 4.61 \\
\hline 0.797037 & 27 & 2398 & 1.104 & 3.66 \\
\hline 0.843455 & 28 & 1854 & 1.061 & 2.83 \\
\hline 0.719670 & 29 & 1394 & 1.001 & 2.13 \\
\hline 0.815027 & 30 & 1082 & 0.906 & 1.65 \\
\hline 0.785026 & 31 & 766 & 0.845 & 1.17 \\
\hline 0.749814 & 32 & 558 & 0.772 & 0.85 \\
\hline 0.710762 & 33 & 348 & 0.822 & 0.53 \\
\hline 0.843007 & 34 & 216 & 0.712 & 0.33 \\
\hline 0.990287 & 35 & 144 & 0.684 & 0.22 \\
\hline 0.892307 & 36 & 74 & 0.636 & 0.11 \\
\hline 0.854608 & 37 & 36 & 0.610 & 0.05 \\
\hline 0.756487 & 38 & 16 & 0.569 & 0.02 \\
\hline 0.735582 & 39 & 0 & - & 0.00 \\
\hline
\end{tabular}

TABLE 5: Combined optimal base (single-digit coefficient, two-digit data).

\begin{tabular}{ccccccc}
\hline$r_{d}$ & $r_{c}$ & Base & Stop band attenuation $(\mathrm{dB})$ & Nonerror-free representations & Worst $\varepsilon$ & \% Nonerror-free \\
\hline 42 & 85 & 0.924440 & $\mathbf{7 5 . 5 0 7 4}$ & 348 & 0.993 & - \\
42 & 85 & 0.871988 & 68.7626 & $\mathbf{0}$ & 0.53 \\
42 & 85 & $\mathbf{0 . 8 1 5 9 5 9}$ & 75.1871 & 88 & 0.756 & 0.00 \\
\hline 42 & 213 & 0.912396 & $\mathbf{7 8 . 2 2 6 5}$ & 196 & 0.839 \\
42 & 213 & $\mathbf{0 . 8 7 2 0 1 8}$ & 77.2010 & $\mathbf{0}$ & - \\
\hline
\end{tabular}

band attenuation. However, comparing the nonerror-free data mapping to Table 3, we can see a large improvement in the representation. This improvement seems to justify the sacrifice of $2 \mathrm{~dB}$ in the stop band.

When considering a hardware implementation, $r_{o}$ will never exceed \pm 255 for the 9-bit system. The 2DLNS-tobinary conversion tables will require $2 r_{o}+2$ entries, one of which is for the zero representation. We will therefore have two inner product CUs each containing tables of 512 entries totaling 1024 entries for both CUs.

\subsubsection{Two-digit coefficients and two-digit data}

We can also apply the blended optimal base to the two-digit coefficient representation as well. Since the ranges on $r_{c}$ are much smaller, we will explore the possibility of having a nonerror-free data representation. As we have seen before, obtaining an error-free data representation will require larger ranges of $r_{d}$ which in turn will require larger tables for the 4 parallel inner product CUs. Table 6 shows various possibilities for $r_{d}$ and $r_{c}$.

Initially 28 and 3 are chosen to maximize the bit width of the product exponent $b_{o}$, but the data representation is poor when the filter's performance is high. As we increment $r_{c}$, we can see an increase of about $0.5 \mathrm{~dB}$ for the best case stop band attenuation. We settle on $r_{c}=5$ as the best case stop band is approximately $80 \mathrm{~dB}$. As we increment $r_{d}$, we see a similar exponential decay trend as before when only optimizing for the data. In the cases of maximum stop band attenuation, the number of nonerror-free representation is quite high. This drops considerably when we sacrifice a little in the stop band $(\sim 0.1 \mathrm{~dB})$. We can begin to reach an error-free data 
TABLE 6: Combined optimal base (two-digit coefficient, two-digit data).

\begin{tabular}{|c|c|c|c|c|c|c|}
\hline$r_{d}$ & $r_{c}$ & Base & Stop band attenuation $(\mathrm{dB})$ & Nonerror-fee representations & Worst $\varepsilon$ & $\%$ Nonerror-free \\
\hline 28 & 3 & 0.895008 & 79.9529 & 23910 & 4.972 & 36.48 \\
\hline 28 & 3 & 0.941001 & 66.9800 & 1908 & 1.000 & 2.91 \\
\hline 28 & 3 & 0.737860 & 77.5315 & 2048 & 1.115 & 3.13 \\
\hline 30 & 4 & 0.858929 & 80.4562 & 4794 & 1.924 & 7.32 \\
\hline 30 & 4 & 0.796725 & 71.2290 & 1062 & 0.932 & 1.62 \\
\hline 30 & 4 & 0.862663 & 75.2993 & 1116 & 0.875 & 1.70 \\
\hline 30 & 4 & 0.745462 & 80.3739 & 1636 & 1.162 & 2.50 \\
\hline 31 & 4 & 0.858929 & 80.4562 & 4398 & 1.924 & 6.71 \\
\hline 31 & 4 & 0.711017 & 69.9018 & 828 & 0.824 & 1.26 \\
\hline 31 & 4 & 0.745462 & 80.3739 & 1534 & 1.162 & 2.34 \\
\hline 33 & 5 & 0.814313 & 80.9394 & 19074 & 3.901 & 29.10 \\
\hline 33 & 5 & 0.764157 & 75.2038 & 394 & 0.802 & 0.60 \\
\hline 33 & 5 & 0.989748 & 75.1037 & 426 & 0.738 & 0.65 \\
\hline 33 & 5 & 0.816596 & 80.5589 & 568 & 0.854 & 0.87 \\
\hline 34 & 4 & 0.858929 & 80.4563 & 3348 & 1.571 & 5.11 \\
\hline 34 & 4 & 0.777919 & 72.8959 & 246 & 0.715 & 0.38 \\
\hline 34 & 4 & 0.777915 & 75.5193 & 284 & 0.693 & 0.43 \\
\hline 34 & 4 & 0.790987 & 79.2613 & 334 & 0.741 & 0.51 \\
\hline 39 & 5 & 0.738251 & 80.9350 & 10806 & 5.512 & 16.49 \\
\hline 39 & 5 & 0.710235 & 76.9878 & 4 & 0.536 & 0.01 \\
\hline 39 & 5 & 0.722788 & 75.8355 & 12 & 0.516 & 0.02 \\
\hline 39 & 5 & 0.732693 & 80.4818 & 24 & 0.570 & 0.04 \\
\hline 40 & 5 & 0.816596 & 80.5634 & 532 & 0.842 & 0.81 \\
\hline 40 & 5 & 0.710131 & 72.6561 & 0 & - & 0.00 \\
\hline 40 & 5 & 0.974939 & 80.2883 & 72 & 0.781 & 0.11 \\
\hline 41 & 5 & 0.915757 & 80.6101 & 738 & 0.854 & 1.13 \\
\hline 41 & 5 & 0.746550 & 77.9541 & 0 & - & 0.00 \\
\hline 41 & 5 & 0.938171 & 80.0786 & 36 & 0.615 & 0.05 \\
\hline 42 & 5 & 0.974939 & 80.4183 & 12 & 0.588 & 0.02 \\
\hline 42 & 5 & 0.944509 & 78.6000 & 0 & - & 0.00 \\
\hline
\end{tabular}

representation when $r_{d}=40$ and above. Depending on the application, a nonerror-free mapping may be acceptable considering the worst case $\varepsilon$ is below 1.0.

\subsubsection{Comparison to the individual optimal base}

When we compare the above results with the previous individual optimal bases, we can see that we have not sacrificed much in terms of stop band attenuation $\left(r_{c}=5\right)$ or exponent ranges for error-free data mapping $\left(r_{d}=40\right)$. This approach seems to offer the best filter performance and data representation as compared to the single-digit coefficients.

For the purposes of implementation, $r_{o}$ will never exceed \pm 45 . We can therefore expect to have four inner product CUs, each of which with 92 entries, totaling 368 entries for four CUs.

\subsection{Comparison of base 3 to the optimal bases}

There are many possibilities available for an optimal base depending on the accuracy required for the filter per- formance and data representation. Table 7 compares the original base 3 and optimal base system's performance to give at least $73 \mathrm{~dB}$ stop band attenuation and a $0 \%$ and $1 \%$ nonerror-free data mapping. For all cases, the optimal base offers saving in the CU LUTs as well as the range of the second-base exponent.

In the single-digit case, we can increase or decrease $r_{d}$ to decrease or increase the nonerror-free representations, respectively.

\subsection{Comparison to general number systems}

We have thus far only shown the improvement in the 2DLNS representation and circuit resources when applying an optimal base compared to the legacy base of 3 . We can further compare the above results with those from common general number systems, such as fixed-point and floating-point binary as well as a fixed-point exponent LNS, which are traditionally used in physical implementations. Table 8 shows a summary of the example filter's performance using these number systems for 1 to 20 bits. Note that the 
TABLE 7: Comparison of standard and optimal base for a minimum $73 \mathrm{~dB}$ system.

\begin{tabular}{|c|c|c|c|c|c|c|}
\hline Base & Stop band attenuation $(\mathrm{dB})$ & $\%$ Nonerror-free & $r_{c}$ & $r_{d}$ & LUT entries per CU & Total LUT entries \\
\hline \multicolumn{7}{|c|}{ Single-digit coefficients } \\
\hline 3.000000 & 73.4312 & 1.00 & 255 & 51 & 614 & 1228 \\
\hline 0.828348 & 73.7272 & 0.67 & 85 & 39 & 250 & 500 \\
\hline Saving & & & & & \multicolumn{2}{|c|}{$59.3 \%$} \\
\hline 3.000000 & 73.4312 & 0.00 & 255 & 93 & 698 & 1396 \\
\hline 0.828348 & 73.7272 & 0.00 & 85 & 45 & 262 & 524 \\
\hline Saving & & & & & \multicolumn{2}{|c|}{$62.5 \%$} \\
\hline \multicolumn{7}{|c|}{ Two-digit coefficients } \\
\hline 3.000000 & 75.4867 & 1.00 & 7 & 51 & 118 & 472 \\
\hline 0.777915 & 75.5193 & 0.43 & 4 & 34 & 78 & 312 \\
\hline Saving & & & & & \multicolumn{2}{|c|}{$33.9 \%$} \\
\hline 3.000000 & 75.4867 & 0.00 & 7 & 93 & 202 & 808 \\
\hline 0.746550 & 77.9541 & 0.00 & 5 & 41 & 94 & 376 \\
\hline Saving & & & & & \multicolumn{2}{|c|}{$53.5 \%$} \\
\hline
\end{tabular}

TABLE 8: Comparison of filter performance for general number systems.

\begin{tabular}{|c|c|c|c|c|c|c|}
\hline \multirow{2}{*}{ Bits } & \multicolumn{2}{|c|}{ Fixed-point binary } & \multicolumn{2}{|c|}{ Floating-point binary } & \multicolumn{2}{|c|}{ LNS with fixed-point exponent } \\
\hline & $\begin{array}{l}\text { Passband ripple } \\
\text { (dB) }\end{array}$ & $\begin{array}{l}\text { Stop band } \\
\text { attenuation } \\
(\mathrm{dB})\end{array}$ & $\begin{array}{l}\text { Passband ripple } \\
\text { (dB) }\end{array}$ & $\begin{array}{l}\text { Stop band } \\
\text { attenuation } \\
(\mathrm{dB})\end{array}$ & $\begin{array}{l}\text { Passband ripple } \\
(\mathrm{dB})\end{array}$ & $\begin{array}{l}\text { Stop band } \\
\text { attenuation } \\
(\mathrm{dB})\end{array}$ \\
\hline 1 & 6.0206 & 6.0206 & 1.4225 & 16.4174 & 2.2454 & 14.6224 \\
\hline 2 & 3.6810 & 9.2326 & 1.2905 & 17.1989 & 1.3542 & 26.0100 \\
\hline 3 & 3.6810 & 9.2326 & 0.1589 & 34.8402 & 0.5313 & 28.6544 \\
\hline 4 & 1.9997 & 13.7377 & 0.1521 & 35.2187 & 0.3403 & 37.1995 \\
\hline 5 & 0.5606 & 24.0824 & 0.0813 & 40.6289 & 0.1493 & 38.1408 \\
\hline 6 & 0.2873 & 29.7543 & 0.0693 & 42.0232 & 0.0776 & 45.6083 \\
\hline 7 & 0.2474 & 31.0320 & 0.0092 & 59.6780 & 0.0422 & 54.1982 \\
\hline 8 & 0.0778 & 41.0001 & 0.0036 & 68.0561 & 0.0249 & 69.0545 \\
\hline 9 & 0.0507 & 44.6930 & 0.0025 & 71.3190 & 0.0127 & 71.2978 \\
\hline 10 & 0.0211 & 52.2642 & 0.0026 & 70.9529 & 0.0064 & 74.0112 \\
\hline 11 & 0.0117 & 57.4244 & 0.0019 & 74.1708 & 0.0032 & 74.8158 \\
\hline 12 & 0.0062 & 62.9227 & 0.0017 & 75.2752 & 0.0017 & 74.7228 \\
\hline 13 & 0.0040 & 66.8129 & 0.0016 & 75.5764 & 0.0012 & 78.0202 \\
\hline 14 & 0.0023 & 71.6093 & 0.0012 & 78.7328 & 0.0009 & 80.0245 \\
\hline 15 & 0.0013 & 76.5792 & 0.0009 & 80.9136 & 0.0008 & 80.8327 \\
\hline 16 & 0.0012 & 77.2776 & 0.0009 & 80.6924 & 0.0008 & 80.9733 \\
\hline 17 & 0.0010 & 78.6727 & 0.0008 & 81.0919 & 0.0008 & 81.0450 \\
\hline 18 & 0.0008 & 80.2013 & 0.0008 & 81.0246 & 0.0008 & 81.1399 \\
\hline 19 & 0.0008 & 80.7061 & 0.0008 & 81.1587 & 0.0008 & 81.1113 \\
\hline 20 & 0.0008 & 81.1363 & 0.0008 & 81.1301 & 0.0008 & 81.1117 \\
\hline
\end{tabular}

bit limitation is applied to the fractional portion of the representations only. The integer portion (i.e., exponent for floating point, integer for LNS exponent) is not considered as it only affects the fixed-point binary representation for values greater than 1; all of the filter's coefficients are however less than 1.

From Table 6, we achieved over $80 \mathrm{~dB}$ stop band attenuation with only 6.6 bits per digit using a two-digit data/coefficient system. In this case only 12 of the 65536 inputs have not any representation error, but it is less than 0.588. The LNS offers the best filter performance with 14 or more bits in the fractional exponent, however this choice may come at the expense of a larger circuit for performing binary-to-LNS-to-binary conversion with 14 fractional bits as well as native LNS addition/subtraction, [10] for example.

In terms of the data representation, all of the mentioned general number system would require at least $14-15$ bits to represent an input integer with $\varepsilon<1$. For this example, 
Table 7 shows that not more than 6.3 bits (per digit) are required when using a two-digit 2DLNS representation $(\varepsilon \leq$ 0.67 ). For each of these general number systems, additional bits in the multipliers (or adders for LNS) will be required to include the calculation of the input data. The presented 2DLNS approach does not need additional precision, but the two-digit system does require an accumulation stage to merge the results from the four separate processing channels.

The aforementioned improvements to 2DLNS with an optimal base as well as a one-bit sign make the architecture more attractive in applications where traditional number systems such as fixed-point and floating-point binary as well as LNS are used. Unfortunately, we are unable to explore a 2DLNS in a wide variety of applications as the choice of the second base is a balance of accuracy and implementation hardware.

\section{EXTENDING TO THREE BASES}

Applying this software to find a set of optimal bases on a multiple base system would have serious scalability issues as we would be performing a linear search geometrically for each base. The complexity of this problem can be considered to be $O\left(n^{k}\right)$ where $n$ is the number of scenarios tested for a single base, and $k$ is the number of nonbinary bases. For the two-digit coefficient case of $r_{d}=40$ and $r_{c}=5, n$ is 20864199. The computational time for any scenario varies considerably with $r_{d}, r_{c}$, and the representation method selected, as they all effect the data generation and search times. We do believe that it is possible to reduce this massive search time for a single-digit 3DLNS system (two nonbinary bases) by merging both algorithmic and range searching methods. By assuming that one of the target representations will have a near zero error, the algorithmic search should provide a series of second bases with the range search supplying the third base. We have yet to attempt this process but we believe it can improve the singledigit representation by using multiple bases with smaller exponent ranges rather than a single base with a larger exponent range. The complexity should be reduced as we are effectively performing many two base optimizations (potentially in parallel) but with a much smaller limitation on the exponents. Using a 3DLNS system with smaller $r$ 's could result in a more compact hardware implementation and better representation than a 2DLNS system.

\section{CONCLUSION}

Since the 2DLNS inner product computational unit requires an LUT for 2DLNS-to-binary conversions, it is important to try to minimize the size of this LUT as the overall system complexity is based on it. We have shown, by example, that selecting an optimal second base can reduce the size of these LUTs and more importantly the range of $b_{o}$, by a minimum of $33 \%$ in one case without impacting the quality of the 2DLNS representation compared to the standard second base of 3. These results should hold true for any other arbitrary second base. As the binary-to-2DLNS conversion hardware also depends on the range of the second-base exponent, selecting an optimal base will offer reductions in this component and may potentially reduce the data paths and storage registers of the complete system. Reducing the range of $b_{o}$ can also impact future architectures, one of which would be a native MDLNS addition/subtraction circuit [11].

By migrating from a two-bit sign to a one-bit sign, the computation time of the optimal base halves and the hardware area and critical paths of published architectures are further reduced as some processing steps are eliminated.

The software for finding an optimal base can be utilized in many different applications other than FIR filter design. The software accepts either a list of real numbers, a range of integers, or both in order to find the best representation with minimal error.

All software, source files, and detailed results can be found at http://research.muscedere.com.

\section{ACKNOWLEDGMENTS}

This work was supported in part by the Natural Sciences and Engineering Council (NSERC) of Canada, and Gennum Corporation. The author would like to acknowledge the important contribution of the Canadian Microelectronics Corporation (CMC) for their equipment, and software loan.

\section{REFERENCES}

[1] V. S. Dimitrov, J. Eskritt, L. Imbert, G. A. Jullien, and W. C. Miller, "The use of the multi-dimensional logarithmic number system in DSP applications," in Proceedings of the 15th IEEE Symposium on Computer Arithmetic, pp. 247-254, Vail, Colo, USA, June 2001.

[2] V. S. Dimitrov and G. A. Jullien, "Loading the bases: a new number representation with applications," IEEE Circuits and Systems Magazine, vol. 3, no. 2, pp. 6-23, 2003.

[3] V. S. Dimitrov, G. A. Jullien, and W. C. Miller, "Theory and applications of the double-base number system," IEEE Transactions on Computers, vol. 48, no. 10, pp. 1098-1106, 1999.

[4] M. G. Arnold, T. A. Bailey, J. R. Cowles, and J. J. Cupal, "Redundant logarithmic arithmetic," IEEE Transactions on Computers, vol. 39, no. 8, pp. 1077-1086, 1990.

[5] E. E. Swartzlander Jr. and A. G. Alexopoulos, "The sign/logarithm number system," IEEE Transactions on Computers, vol. C-24, no. 12, pp. 1238-1242, 1975.

[6] R. Muscedere, V. Dimitrov, G. Jullien, and W. Miller, "A low-power two-digit multi-dimensional logarithmic number system filterbank architecture for a digital hearing aid," EURASIP Journal on Applied Signal Processing, vol. 2005, no. 18, pp. 3015-3025, 2005.

[7] R. Muscedere, V. Dimitrov, G. A. Jullien, and W. C. Miller, "Efficient techniques for binary-to-multidigit multidimensional logarithmic number system conversion using rangeaddressable look-up tables," IEEE Transactions on Computers, vol. 54, no. 3, pp. 257-271, 2005.

[8] H. Li, G. A. Jullien, V. S. Dimitrov, M. Ahmadi, and W. C. Miller, "A 2-digit multidimensional logarithmic number system filterbank for a digital hearing aid architecture," in Proceedings of the IEEE International Symposium on Circuits and Systems (ISCAS '02), vol. 2, pp. 760-763, Scottsdale, Ariz, USA, May 2002. 
[9] J. Eskritt, R. Muscedere, G. A. Jullien, V. S. Dimitrov, and W. C. Miller, "A 2-digit DBNS filter architecture," in Proceedings of IEEE Workshop on Signal Processing Systems (SiPS '00), pp. 447-456, Lafayette, La, USA, October 2000.

[10] D. M. Lewis, "An architecture for addition and subtraction of long word length numbers in the logarithmic number system," IEEE Transactions on Computers, vol. 39, no. 11, pp. 13251336, 1990.

[11] R. Muscedere, "A hardware efficient single-digit 2dimensional logarithmic number system addition and subtraction circuit," submitted to IEEE Transactions on Circuits and Systems. 